\title{
Microfilaria malayi in uveitis: Case report
}

\author{
K. ANANDAKANNAN AND C. P. GUPTA
}

SUMMARY A case of uveitis with microfilariae in the anterior chamber is reported. The organism was recovered from the aqueous and identified as Brugia malayi

The first recorded case of an intraocular filaria was published by Mercier (1771), and numerous cases have since been reported. Here we present a case of unilateral uveitis with the presence of microfilaria in the anterior chamber confirmed by aqueous puncture and microscopy as Brugia malayi.

\section{Case history}

The patient, a man aged 60 years, came from a place endemic for filariasis and reported to the Government Ophthalmic Hospital on 14 July 1976 complaining of redness and watering in the right eye of 1 week's duration. It was acute in onset and he had had no previous attacks. There was no history of having had fever with rigor suggestive of filariasis.

On general examination there was no lymphadenopathy; the inguinal group of glands especially were normal. There was a minimal hydrocoele of the scrotum on both sides. Examination of the right eye showed swelling of the lid and ciliary injection. The cornea was clear. The pupil was small and reacted sluggishly to light. A posterior cortical cataract was present. The right vision was $6 / 60$. The fundus view was hazy but the disc appeared normal. Slit-lamp examination showed no KPs but a marked aqueous flare and many cells in the anterior chamber.

Subsequent slit-lamp examination done a week after admission showed the presence of a few actively motile organisms in the anterior chamber. They were seen only in the pupillary area and appeared to be attached to the anterior lens capsule. The intensity of the flare and cells remained the same. The patient was put on routine therapy for uveitis. The aqueous was tapped and a drop examined beneath a cover slip under the microscope.

Actively motile organisms were found to be swarming in the field. Paracentesis was repeated after 3 days and the same type of organisms were found to be present in the aqueous. However,

Address for reprints: Dr K. Anandakannan, 7 Marshall's Road, Egmore, Madras 600008, India attempts to stain the organisms were unsuccessful. Photographs of the organisms could not be made owing to the poor background despite several attempts.

\section{Investigations}

Urine: albumin, sugar-nil. Mantoux test-positive. Blood VDRL - positive in 1 dilution. Total white cell count- $8.2 \times 10^{9} / 1$; polymorphs $50 \%$, eosinophils $12 \%$, lymphocytes $38 \%$. ESR -0.5 hour $5 \mathrm{~mm}-1$ hour $10 \mathrm{~mm}$.

Night blood was positive for microfilaria. A smear was done and stained with iodine, which showed the presence of microfilariae. Owing to the presence of nuclei up to the tail tip and its bifurcation at the tip it was identified as the genus Brugia malayi (Figs. 1 and 2). The patient was started on diethylcarbamazine systemically $2 \mathrm{mg} / \mathrm{kg}$ body weight for 15 days. Locally he had atropine and cortisone applied thrice daily. The eye became quiet in 1 week and slit-lamp examination done after 2 weeks showed no flare or cells; a few microfilariae were still seen in the anterior chamber, but they were fewer in number and less motile. Subsequently, the patient was discharged and advised to come after a month for follow-up.

\section{Discussion}

The filariae are parasites belonging to the group of nematodes. Four types are pathogenic to man; Wuchereria bancrofti, causing filariasis; Onchocerca volvulus, causing onchocerciasis; Loa loa, causing loaiasis; and Dracunculus medinensia, causing dracunculiasis. Filariasis is transmitted by a mosquito which harbours the larvae and microfilariae. Five types of microfilaria are found in blood. Mf. bancrofti, Mf. malayi, and $M f$. loa, which are sheathed and have a periodicity. Mf. perstans and $M f$. ozzardi are non-sheathed and non-periodic. $M f$. bancrofti and malayi are common in India.

Brugia malayi can occur alone or in conjunction 


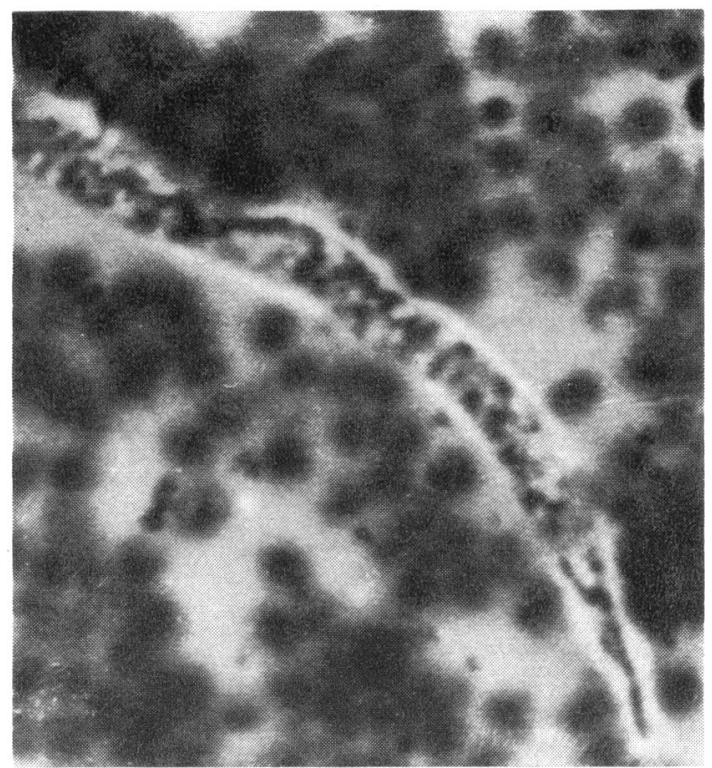

Fig. 1 Showing the tail of the parasite with birfurcated nucleus

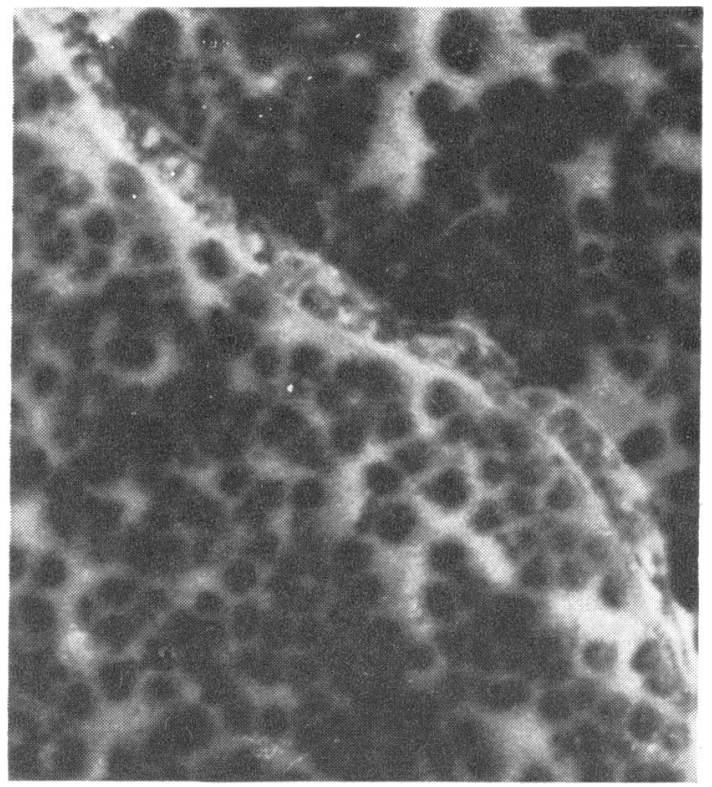

Fig. 2 The parasite seen under the low power microscope

with bancroftian filaria. The adult worm is found in the lymphatic system; microfilariae are found in the peripheral blood at night. They show the following peculiarities: (1) Small in size $(230 \times 6 \mu \mathrm{m})$;

(2) secondary kinks instead of smooth curves; (3) double stylets at anterior end; (4) cephalic space broad; (5) tail tip not free from nuclei.
The intermediate hosts are various species of Mansonioides and certain species of Anopheles mosquito. Domestic animals, cats, and dogs may serve as reservoirs of infection (Chatterjee, 1971).

The ocular manifestations of filariasis are elephantiasis of the eyelids; iritis caused by the adult worm; retinal haemorrhages or the presence of microfilariae in the lachrymal gland secretion (Toussaint and Davis, 1965). The microfilariae liberated from the adult worm inhabiting the lymphatics of the pelvis and lower part of the abdomen enter the venous system through the thoracic duct (Jones et al., 1938). After going through the pulmonary circulation they enter the systemic circulation. Entry into the anterior chamber may be through the ciliary vessels.

The presence of an adult worm in the anterior chamber producing iritis has been reported by many authors (Nayar and Pillai, 1932; Wright, 1934). Sun (1958) in China observed microfilariae swimming actively in the anterior chamber. With diethylcarbarmazine and carbosone the microfilariae disappeared from the blood in 1 month and from the anterior chamber in 4 months (Duke-Elder, 1965). Probably this is the first report from India in which microfilariae have been seen and isolated from the anterior chamber.

Living microfilariae in the blood do not produce any pathogenic effect. But in this case, as the routine investigations were negative and the uveitis responded to diethylcarbamazine it may be a rare case of uveitis caused by the microfilariae in the anterior chamber.

We hereby acknowledge our thanks to the Superintendent, Government Ophthalmic Hospital, Madras, for having permitted us to publish this article. We also thank Dr Subramanian, Professor of Bacteriology, Madras Medical College, who helped in identifying the microfilariae.

\section{References}

Chatterjee, K. D. (1971). Parasitology, 7th edn, p. 183. K. D. Chatterjee: Calcutta.

Duke-Elder, S. (1965). System of Ophthalmology, Vol. 8, p. 401. Kimpton: London.

Duke-Elder, S. (1966). System of Ophthalmology, Vol. 9, p. 453. Kimpton: London.

Jones, T., Jordan, L. W., and Sullivan, N. P. (1938). Archives of Ophthalmology, 20, 1006.

Mercier (1771) quoted in Parsons, J. H. (1904). Pathology of the Eye, Vol. 1, p. 321. Hodder \& Stoughton: London.

Nayar, K. K., and Pillai, A. K. (1932). British Journal of Ophthalmology, 16, 549 .

Sun, K. Y. (1958). Chinese Medical Journal, 77, 74

Toussaint, D., and Danis, P. (1965). Archives of Ophthalmology, 74, 470 .

Wright, R. E. (1934). British Journal of Ophthalmology, 18, 646. 